\title{
POSSIBLE MECHANISM OF ACTION OF MENTHA ARVENSIS IN CARDIOVASCULAR DISEASES
}

\author{
Saima Gul ${ }^{1}$, Humaira Gul' \& Rukhsana Nawaz \\ 1. PAPRSB Institute of Health Sciences, University Brunei Darussalam, \\ 2. Department of Botany, Abdul Wali Khan University Mardan, Pakistan, \\ 3. Institute of Biological and Pharmaceutical Sciences, Dow University of Health Sciences, Karachi, Pakistan \\ Correspondence Author Email: saimaa.gul@gmail.com
}

\begin{abstract}
Mentha arvensis (MA) is traditionally used in hypertension and in patients with ischemic heart disease. The scientific rationale for its use is not known. In order to find a pharmacological basis of its use in traditional medicine we made three polarity based fraction from the crude extract of MA and investigated their effects on arachidonic acid metabolism. MA inhibited arachidonic acid metabolite thromboxane B2-a stable analogue of thromboxane-A2, formed via cyclooxygenase pathway and lipoxygenase product 1 and 12-hydroxyeicosatetraenoic acid formed via lipoxygenase pathway. More potent inhibition of thromboxane-B2 compared to lipoxygenase product 1 and 12-hydroxyeicosatetraenoic acid was observed and indicates MA might possess antiplatelet activity as thromboxane-B2 is one of the strongest proponents of platelet aggregation. When MA was investigated for antiplatelet activity, it was found to inhibit human platelet aggregation induced by arachidonic acid as well as by adenosine diphosphate and platelet activating factor while collagen-induced platelet aggregation was unaffected by MA. Since arachidonic acid-induced aggregation is mediated through thromboxane-A2, these results indicate that inhibition of platelet aggregation may be responsible for the observed beneficial effects of MA in patients with ischemic heart disease. Furthermore, MA was also effective in enhancing glutathione peroxidase activity, although it had no significant effect on superoxide dismutase activity. These activities were however, distributed throughout various fraction of MA.
\end{abstract}

\section{KEYWORDS}

Mentha arvensis, Inflammation, Platelet aggregation, Thromboxane, Cyclooxygenase, Lipoxygenase

\section{INTRODUCTION}

Mentha arensis (MA or wild mint) is a common edible aromatic perennial herb, whose leaves are universally used for flavouring foods and beverages (Sharma, 2002). It belongs to genus Lamiaceae which comprises approximately 25 - 30 species which may be found in temperate regions of Eurasia, Australia, and South Africa (Ali, 2001). Although this is a distinct genus, it is notorious for its long history of cultivation, naturalization, and vegetative plasticity (Ali, 2001). Members of the genus are characterized by their volatile oils which are of great economic importance, being used by the flavor, fragrance, and pharmaceutical industries. A review of the literature reveals that the aerial materials of some members are used for herbal teas and condiments (Basuer, 1999), as spasmolytics, antibacterial agents, and promoters of gas secretion (Tyler, 1993), and for their analgesic and antigenotoxic properties (Sylianko, 1986). Several medicinal properties have been ascribed to it (Chopra, 1956; Aswal, 1984) including abortifacient and antinidational properties in various mammalian species (Bodhankar, 1971; Kanjanapothi, 1981; Satyavati, 1987; Garg \& Jacob, 1994). Aqueous extract of MA leaves can effectively suppress male fertility also, if administered via intramuscular injections just for 20 days (Sharma, 1996, 2002). Since MA is traditionally used in hypertension and in patients with ischemic heart disease, we hypothesized that it might interfere with cyclooxygenase (COX) and lipoxygenase (LOX) enzymes which are primary targets of many cardiovascular and anti-inflammatory drugs. Furthermore, a number of previous studies (Zia-Ul-Haq, 2012; Hussain, 2010 \& Imran, 2012) report that plants used in traditional cardiovascular medicine possess activities against arachidonic acid (AA) metabolism and platelet aggregation. We therefore, investigated the effect of MA on arachidonic acid metabolism and platelet aggregation.

Furthermore, the effects of MA were also observed on the activities of antioxidant enzymes superoxide dismutase (SOD) and glutathione peroxidase (GPx).

\section{MATERIAL AND METHODS}

All organic solvents, chemicals and reagents used in the experiments were of analytical and highest purity grade. All the chemicals are purchased from Sigma chemical company, St. Louis, USA except $14 \mathrm{C}$ arachidonic acid which was purchased from Amersham Biosciences; acetic acid and citric acid from BDH, sodium phosphate (mono and dibasic) from Merck and m-3M3FBS was purchased from Calbiochem (La Jolla, CA, USA).

\section{EXTRACTION OF PLANT MATERIAL}

Fresh leaves of MA (4 kg) were collected in Kohat District, Khyber Pakhtunkhwa, Pakistan, and authenticated by an Assistant Professor at the Department of Botany, Abdul Wali Khan University Mardan, Pakistan. The leaves of MA were shade dried and then ground using a commercial grinder to obtain fine powder. Nine hundred (900) grams of ground material was soaked in 5 liter aqueous methanol and left for 24 hours at $4{ }^{\circ} \mathrm{C}$. The extract was filtered using a filtration flask and whattmann paper. This extract was evaporated on a rotary evaporator under reduced pressure ($760 \mathrm{mmHg}$ ) to a thick, semi-solid mass of dark brown color i.e. crude extract. 


\section{FRACTIONATION OF THE CRUDE EXTRACT}

Fractionation of the crude extract was done following the procedure described previously (Ahmed, 2013) Two hundred grams of the extract was dissolved in distilled water. This was then introduced in a separating funnel. n-hexane $(50-70 \mathrm{ml})$ was then added into the same separating funnel. This mixture was shaken vigorously, regularly allowing the air to escape out. It was kept for about $30 \mathrm{~min}$ to let the two layers separate. The upper layer of $\mathrm{n}$ hexane was acquired and the same procedure was repeated twice and all the n-hexane layers were collected and concentrated in a rotary evaporator to obtain the $\mathrm{n}$-hexane fraction. Chloroforml (50 $\mathrm{ml}$ ) was then added to the remaining layer and the same process was repeated as with $\mathrm{n}$-hexane, finally obtaining the chloroform fraction, the yield of both fractions was $23 \%$ and $17 \%$, respectively, while the remaining layer was filtered using a filtration flask and whattmann paper and used in the experiments as aqueous fraction.

\section{ARACHIDONIC ACID METABOLISM BY HUMAN PLATELETS}

Archidonic acid metabolism and thromboxane B2 (TXB2) formation was studied using a Berthold T.L.C. linear analyzer and chromatography data system (Model LKB 511, Berthold, W. Germany) as described previously (Saeed, 2007). Human blood platelets were obtained in plastic bags containing 30-40 ml concentrated platelet rich plasma (PRP) from the diagnostic laboratory of the Aga Khan University, Karachi. The PRP was centrifuged at $1200 \mathrm{~g}$ for $20 \mathrm{~min}$ and the sedimented platelets were washed twice with an ice-cold phosphate buffer (50 mM, PH 7.4), containing $\mathrm{NaCl}(0.15 \mathrm{M})$ and EDTA $(0.2 \mathrm{mM})$. After centrifugation platelets were re-suspended in the same buffer without EDTA at the initial PRP concentration. The PRP suspension was homogenized at $4{ }^{\circ} \mathrm{C}$ using a polytron homogenizer for $15 \mathrm{sec}$ and the homogenate centrifuged at $1200 \mathrm{~g}$ for $20 \mathrm{~min}$. Supernatant ( $300 \mu$ l containing $0.4 \mathrm{mg}$ protein) was incubated with $10 \mu \mathrm{g}$ unlabelled AA and $0.1 \mu \mathrm{Ci}$ [1-14C]AA in the presence and absence of test fraction. After 15 min of gentle shaking in air at 37 ${ }^{\circ} \mathrm{C}$ the reaction was stopped by adding $0.4 \mathrm{ml}$ citric acid $(0.4 \mathrm{M})$ and ethyl acetate $(7.0 \mathrm{ml})$. After mixing and centrifuging at $600 \mathrm{~g}$ for $5 \mathrm{~min}$ at $4{ }^{\circ} \mathrm{C}$, the organic layer was separated and evaporated to dryness under nitrogen. Residues were dissolved in $40 \mu 1$ of ethanol and $20 \mu \mathrm{l}$ was applied to silica gel $\mathrm{G}$ thin layer chromatography (TLC) plates (Analtech Delaware, USA). The AA, TXB2 (a stable degradation product of TXA2), lipoxygenase product 1 (LP1) and 12-hydroxy eicosatetraenoic acid (12-HETE) standards were plotted separately.

\section{SOLVENT SYSTEMS FOR DEVELOPING TLC PLATES}

The TLC plates were developed in ether/petroleum ether [boiling range 40-60] /acetic acid (50:50:1, v/v) to a distance of $17 \mathrm{~cm}$. By use of this solvent system the various lipoxygenasse products such as 12-HETE were separated with TXB2 and prostaglandins remaining at the origin. The solvent system used for the separation of TXB2 was ethyl acetate/iso-octane/water/acetic acid (11:5:10:2, $\mathrm{v} / \mathrm{v}$ upper phase). Radioactive zones were located and quantified by use of a Berthold TLC linear analyzer and chromatography data system (Model LKB 511, Berthold, Germany).

\section{PREPARATION OF PLATELETS}

Platelet effects were studied in human platelets by taking blood via venipuncture from normal human volunteers reported to be free of medication for 7 days. Blood sample were mixed with $3.8 \%$ (w/v) sodium citrate solution (9:1) and centrifuged at $260 \times \mathrm{g}$ for $15 \mathrm{~min}$ at $20{ }^{\circ} \mathrm{C}$ to obtain PRP. The remaining blood samples were centrifuged at $1200 \times \mathrm{g}$ for $10 \mathrm{~min}$ to obtain platelet-poor plasma (PPP). Platelet count was determined by phase contrast microscopy and all aggregation studies were carried out at $37{ }^{\circ} \mathrm{C}$ with PRP having platelet counts between 2.5 and $3.0 \times 108 \mathrm{ml}-1$ of plasma.

\section{MEASUREMENT OF PLATELET AGGREGATION}

Aggregation was monitored using dual-channel Lumiaggregometer (Model 400 Chronolog Corporation, Chicago, USA) using $0.45 \mathrm{ml}$ aliquots of PRP (Aslam, 2008; Saeed, 2006). The final volume was made up to $0.5 \mathrm{ml}$ with the test fraction. Aggregation was induced by AA (1.7 $\mathrm{mM})$, adenosine diphosphate (ADP) $(2.2 \mu \mathrm{M})$, platelet activating factor (PAF) $(0.8 \mu \mathrm{M})$, collagen. The antiplatelet effects of test fractions were studied by pretreatment of PRP with each fraction for 2 min followed by addition of platelet agonist. The resulting aggregation was recorded for $5 \mathrm{~min}$ after challenge by the change in light transmission as a function of time. After establishing the antiplatelet activity against various agonists, dose-response curves were constructed to calculate the $1 \mathrm{C} 50$ values.

\section{GLUTATHIONE PEROXIDASE ACTIVITY}

GPx levels were determined using commercially available kits from RANDOX, UK and assays were carried out using a spectrophotometer DU 800 (Beckmann, USA). After adding all the chemicals, crude extract and different fractions obtained were added before the addition of substrate (i.e. t-Butyl-hydroperoxide). The absorbance at $340 \mathrm{~nm}$ was measured. GPx was measured by coupling the peroxidase reaction with the reduction of oxidized glutathione by glutathione reductase and the reduced form of nicotinamide adenine dinucleotide phosphate (NADPH) (18). Reduction of the substrate was followed by the decrease in absorbance of NADPH at $340 \mathrm{~nm}$. Activity was evaluated using glutathione as the co-substrate (Gul, 2011).

\section{SUPEROXIDE DISMUTASE ACTIVITY}

SOD activity was determined using the same commercially available kits and spectrophotometer. SOD activity assay was conducted as described previously (Gul, 2013). After adding all the chemicals, different fractions were added before the addition of substrate i.e. 2-(4-iodophenyl)-3-(4-nitrophenol)-5phenyltetrazolium chloride (INT) to obtain a formazane dye. The absorbance at $505 \mathrm{~nm}$ was measured. The method involved xanthine and xanthine oxidase to generate superoxide radicals. The SOD activity was measured taking into consideration the degree of inhibition in this reaction. One unit of SOD can cause 50\% inhibition of the rate of reduction of the INT under the condition of the assay. 


\section{RESULTS}

Crude extract of MA inhibited COX-mediated pathway of AA metabolism more potently compared to LOX-mediated pathway. IC50 (mean \pm SD in $\mu \mathrm{g}$ ) values were $22 \pm 6$ for TXB2, $92 \pm 14$ for LP1 and $104 \pm 21$ for 12-HETE. Crude extract of MA did not inhibit collagen-induced platelet aggregation (figure 1D); however, PAF and ADP-induced aggregations were weakly inhibited while the most potent antiplatelet effect was observed against AAinduced-platelet aggregation. The dose of the extract showing 50\% inhibitory effect against platelet aggregation (IC50 value in $\mu \mathrm{g}$ ) against various agonists were $19 \pm 4$ for AA, $71 \pm 12$ for ADP,
$113 \pm 22$ for PAF and $14 \pm 3$ for aspirin. Crude extract was able to enhance the activity of GPx (EC50, 32 \pm 6 ), however, it had no significant effect on the activity of SOD up to a dose of $1 \mathrm{mg}$ (table 1).Aqueous fraction of MA inhibited COX-mediated production of TXB2 (IC50, 28 $\pm 5 \mu \mathrm{g}$ ) but was slightly less potent compared to crude extract while no inhibition of LOX-mediated pathway was seen up to $1 \mathrm{mg}$. Aqueous fraction of MA potently inhibited AAinduced platelet aggregation (IC50, 29 $\pm 6 \mu \mathrm{g}$ ) (figure 1A) while no significant antiplatelet action was observed against ADP, PAF and collagen-induced platelet aggregation up to $1 \mathrm{mg}$. Aqueous fraction had no appreciable effect on the activities of GPx and/or SOD up to a dose of $1 \mathrm{mg}($ table 1$)$.
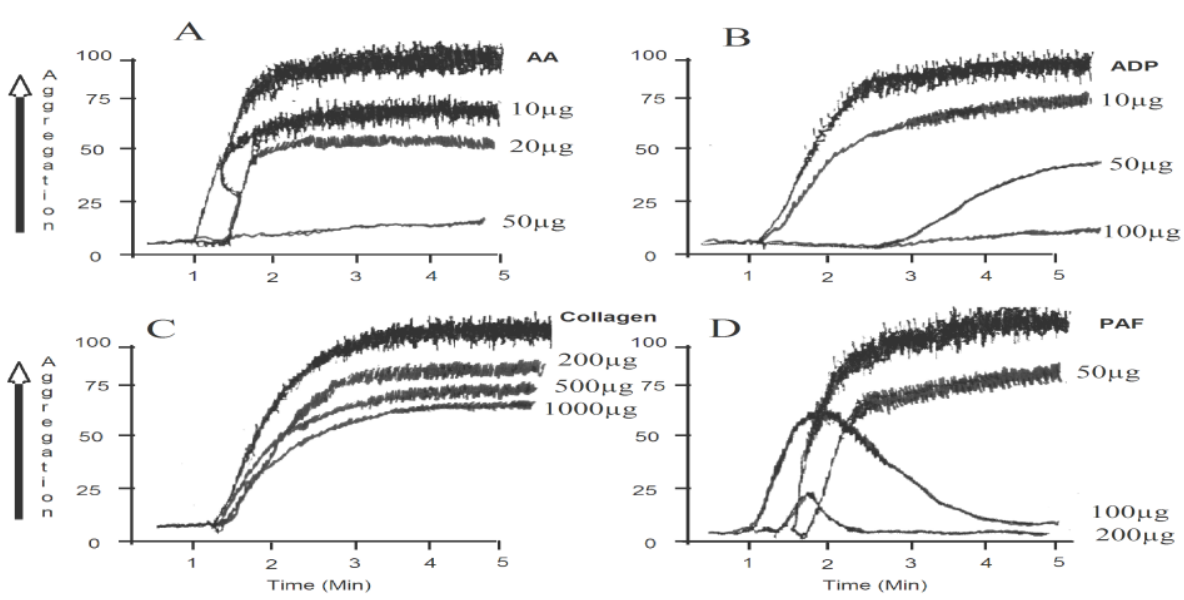

Figure 1: Representative scans of the pharmacological actions of the crude extract of $\mathrm{MA}$ and its various fractions on platelet aggregation induced by a diversity of platelet agonists, A (aqueous fraction on AA), B (chloroform fraction on ADP), C (n-hexane fraction on PAF) and D (crude extract on collagen), $n=5$.

Chloroform fraction of MA had no significant inhibition of TXB2, 12-HETE and LP-1 up to a dose of 1mg. Chloroform fraction of MA was also ineffective against AA and collagen induced platelet aggregation, weakly active against PAF-induced platelet aggregation while its most potent inhibition was seen against ADPinduced platelet aggregation (figure 1B). Chloroform fraction had no substantial effect on SOD activity up to a dose of $1 \mathrm{mg}$ but elevated GPx activity potently with EC50, $38 \pm 5 \mu \mathrm{g}$ (table 1). nhexane fraction of MA potently inhibited LOX-mediated production of LP-1 and 12 HETE (IC50, 34 $\pm 5 \mu \mathrm{g}$ and $29 \pm 3 \mu \mathrm{g}$ respectively) (figure 2) but no significant effect was observed on COX-mediated pathway of AA metabolism. n-hexane fraction of MA weakly inhibited PAF (figure 1C) and AA-induced platelet aggregation (IC50, $82 \pm 15 \mu \mathrm{g}$ and $91 \pm 14 \mu \mathrm{g}$ respectively) but no significant protection was offered against collagen and ADP induced platelet aggregation up to $1 \mathrm{mg}$. $\mathrm{n}$-hexane fraction was the most potent in enhancing GPx activity (EC50, 21 $\pm 3 \mu \mathrm{g}$ ), but no significant effect on SOD activity was observed (table 1).

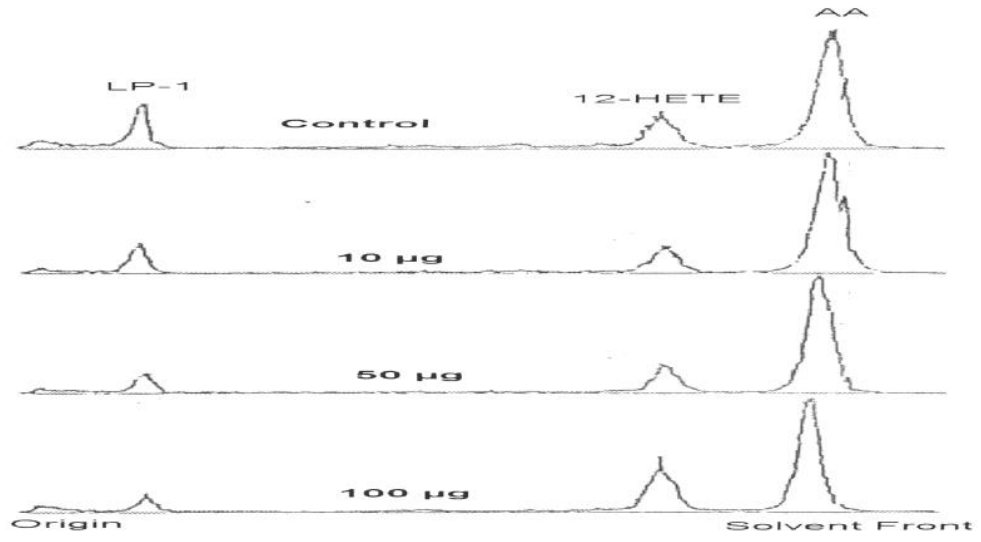

Figure 2: Representative scan of the effects of the $n$-hexane fraction of MA on AA metabolism through LOX pathway, $n=6$. 


\begin{tabular}{|l|l|l|l|l|l|}
\hline No & Assay & Crude & Aqueous & Chloroform & n-Hexane \\
\hline 1 & PAF & $113(22)$ & $1 \mathrm{mg}$ & $67(12)$ & $82(15)$ \\
\hline 2 & AA & $19(4)$ & $29(6)$ & $1 \mathrm{mg}$ & $91(14)$ \\
\hline 3 & Collagen & $1 \mathrm{mg}$ & $1 \mathrm{mg}$ & $1 \mathrm{mg}$ & $1 \mathrm{mg}$ \\
\hline 4 & ADP & $71(12)$ & $1 \mathrm{mg}$ & $34(6)$ & $1 \mathrm{mg}$ \\
\hline 5 & LP-1 & $92(14)$ & $1 \mathrm{mg}$ & $1 \mathrm{mg}$ & $34(5)$ \\
\hline 6 & $12-$ HETE & $104(21)$ & $1 \mathrm{mg}$ & $1 \mathrm{mg}$ & $29(3)$ \\
\hline 7 & TXB2 & $22(6)$ & $28(5)$ & $1 \mathrm{mg}$ & $1 \mathrm{mg}$ \\
\hline 8 & GPx (EC50) & $32(6)$ & $1 \mathrm{mg}$ & $38(5)$ & $21(3)$ \\
\hline 9 & SOD (EC50) & $1 \mathrm{mg}$ & $1 \mathrm{mg}$ & $1 \mathrm{mg}$ & $1 \mathrm{mg}$ \\
\hline
\end{tabular}

Table 1: IC50 values of the crude extract of MA and its various fractions. 1mg indicates that no significant effect was observed up to a dose of $1 \mathrm{mg}$. All the other values are given as mean (SD) in $\mu \mathrm{g}$.

\section{DISCUSSION}

Plants have been used for centuries to obtain treatments for various human diseases (Zia-Ul-Haq, 2010; Ahmad, 2010). There are reports suggesting natural inhibition of AA metabolites (Saeed, 2003). Dual inhibition of LOX and COX pathways by natural compounds is also known and thought to be contributed by the secondary metabolism of plants (Saeed, 2004). Natural inhibitors of both inflammation and oxidative stress, however, are less common but could be of great therapeutic importance. Here in this report, we show that MA strengthens the antioxidant defense system and inhibits LOX and COX pathways of AA metabolism.COX is the enzyme that catalyzes the rate-limiting step in prostaglandin synthesis, converting AA into prostaglandin $\mathrm{H} 2$, which is then further metabolized to prostaglandin E2, TXA2 and other eicosanoids (Ahmed, 2014). COX have two isoforms, COX-1 and COX-2, both of which are implicated in disease and their inhibition has been shown to benefit in cardiovascular diseases including myocardial infarction (Saeed and Ahmed 2005, 2006). Potent inhibition of AA-induced-platelet aggregation and COX metabolite TXB2 by the crude extract of MA suggests the presence of strong inhibitory compounds against COX pathway of AA metabolism Crude extract was also found potent in elevating GPx activity but lack of action on SOD suggests that MA may not possess compounds affecting SOD. Weak inhibition of LOX metabolites suggested presence of LOX inhibitory compounds in low quantity. These findings are important in relation to the facts that COX metabolites have been implicated in the pathophysiology of cardiovascular disease, cancer, and inflammatory diseases. Nonsteroidal anti-inflammatory drugs (NSAIDs) such as aspirin, acetaminophen, ibuprofen, indomethacin, and naproxen directly target COX.

LOX is an important enzyme involved in AA metabolism and its metabolites are implicated in a number of diseases. In the present study crude extract of MA was partially effective against 12-LOX metabolites LP1 and 12-HETE, however, potent effect was observed with n-hexane fraction. Aqueous and chloroform fractions did not show significant effect up to $1 \mathrm{mg}$. This indicated the presence of 12-LOX inhibitory components in the $\mathrm{n}$-hexane fraction of MA and indicates their non-polar nature. Platelettype12-LOX have been found in different cancer tissues, including melanoma, prostate and epidermal cancers (Steele, 1999). Evidence indicates that 12-LOX is involved in both cancer cell proliferation and survival (Yamamoto, 1999). Inhibition of 12LOX with either 12-LOX inhibitors or a 12-LOX antisense oligonucleotide inhibits proliferation and induces apoptosis in carcinosarcoma cells, while adding back the 12-LOX metabolite, 12(S)-HETE prevents 12-LOX inhibitor-induced apoptosis (Tang, 1997). Similar activities have been reported for other plans as well.

Platelets play a critical role in the initial restriction of blood loss following vascular injury by rapidly adhering to exposed subendothelial matrix components and aggregating to form a provisional plug. When crude extract of MA was tested against platelet aggregation induced by AA, ADP, PAF and collagen, it partially inhibited PAF and ADP but potently blocked AA-induced platelet aggregation, while no antiplatelet effect was observed against collagen. Aqueous fraction showed potent activity against AA but was ineffective against all other platelet agonists. Chloroform was the most potent fraction against ADP and partially active against PAF while it was devoid of any significant action against remaining platelet agonists. N-hexane fraction was only weakly active against $\mathrm{AA}$ and $\mathrm{PAF}$ and inactive against $\mathrm{ADP}$ and collagen. These findings suggest that relatively polar nature of the antiplatelet compounds of MA because potent antiplatelet effects were observed in aqueous and chloroform fractions compared to the n-hexane fraction. Similar platelet inhibitory activities have been reported by a number of other investigators (Zia-Ul-Haq, 2012; Bukhari, 2010 \& Hussain, 2009). 
In conclusion our study shows that of MA possesses strong inhibitory activity against COX and LOX-mediated metabolites and potent antiplatelet activtiy against AA and ADP, with partial activity againt PAF and no activity against collagen. Furthermore, no effect was observed on SOD activity while GPx activity was elevated significantly. The pattern of inhibition by various fractions suggest that activity against TXB2 as well as antiplatelet effect against AA was concentrated in aqueous fraction suggesting the polar nature of compounds responsible for these ffects. LOXinhibitory activity was primarily found in $n$-hexane fraction suggesting their non-polar character. Although no effect was observed on the activity of SOD, GPx activity was significantly enhanced by crude extract as well as by chloroform and n-hexane fractions. Finally antiplatelet activity against ADP was concentrated in the chloroform fraction. Since no effective inhibitor is currently available to block the COX and LOX pathways, and simultaneously enhancing the GPx activity, various fractions of MA might be valuable for the isolation of pure compounds that show dual inhibition of COX and LOX pathways. This may prove to be extremely useful for treatment and prevention of a number of inflammatory conditions.

\section{REFERENCES}

- Ahmad, S., Akhtar, M., Zia-ul-Haq, M., Mehjabeen and Ahmed, S. (2010). Antifungal and nematicidal activity of selected legumes of Pakistan. Pakistan Journal of Botany, 42(2): 1327-1331.

- Ahmed, S., Gul, S., Gul, H., Bangash, M.H. (2013). Dual Inhibitory Activities of Adhatoda Vasica against Cyclooxygenase and Lipoxygenase. International Journal of Endorsing Health Science Research, 1 (1): 14-17.

- Ahmed, S., Gul, S., Gul, H., Bangash, M.H. (2013). Antiinflammatory and Anti-platelet Activities of Avena sativa are mediated through the inhibition of Cyclooxygenase and Lipoxygenase Enzymes International Journal of Endorsing Health Science, 1(2):62-65.

- $\quad$ Ahmed, S., Gul, S., Zia-Ul-Haq, M., Stanković, M.S. (2014). Pharmacological basis of the use of Acorus calamus L. in inflammatory diseases and underlying signal transduction pathways. Latin American and Caribbean Bulletin of Medicinal and Aromatic Plants, 13(1): 38-46.

- Ali, M.S., Saleem, M., Ahmad, W., Parvez, M., Yamdagni, R. (2002). A chlorinated monoterpene ketone, acylated âsitosterol glycosides and a flavanone glycoside from Mentha longifolia (Lamiaceae). Phytochemistry, 59: 889-895.

- Aswal, B.S., Bhakuni, D.S., Goel, A.K., Kar, K., Mehrotra, B.N. (1984). Screening of Indian plants for biological activity, Part XI. Indian Journal of Experimental Biology, 22: 287-492.

- Aslam, R., Saeed, S.A., Ahmed, S., Connor, J.D. (2008). Plasma lipoproteins inhibit platelet aggregation and arachidonic acid metabolism in experimental hypercholesterolemia. Clinical and Experimental Physiology and Pharmacology, 35(5-6):656-62.

- Basüer, K.H.C. and Kurkcuoglu. (1999) M. Essential oils of Mentha species from Northern Turkey. J. Essent. Oil Res. 11: 579-588.

- Bodhankar, S.L., Garg, S.K., Mathur, V.S. (1971). Effect of Mentha ar_ensis Linn on fertility in female albino rats. Bulletin PGI, 5: 66-68.
- Bukhari, I.A., Khan, R.A., Gilani, A.H., Ahmed, S., Saeed, S.A. (2010). Analgesic, anti-inflammatory and anti-platelet activities of the methanolic extract of Acacia modesta leaves. Inflammopharmacology, 18(4):187-96.

- Chopra, R.N., Nayar, S.L., Chopra, I.C. (1956). Glossary of Indian Medicinal Plants. Publication \& Information Department, CSIR New Delhi.

- Garg, P., Jacob, D. (1994). Mentha arvensis leaf: an effective antinidational substance in laboratory mouse. Journal of Advanced Zoology, 15: 32-36.

- Gul, S., Ahmed, S., Gul, H., Kaneez, K.F. (2011). Investigating the Protective Effect of Solanum melongena. Asian Journal of Health, 1(1):4-9243.

- Gul, S., Ahmed, S., Gul, H., Shad, K.F., Zia-Ul-Haq, M., Badiu, D. (2013). The antioxidant potential of Brassica rapa L. on glutathione peroxidase, superoxide dismutase enzymes and total antioxidant status. Revista Română de Medicină de Laborator, 21 (2/4):161-169

- Hussain, J., Jamila, N., Gilani, S.A., Abbas, G., and Ahmed, S. (2009). Anti-Platelet aggregation, antiglycation, cytotoxic, phytotoxic and antimicrobial activities of extracts of Nepeta juncea. African Journal of Biotechnology, 8(6): 935-940.

- Hussain, J., Khan, F.U., Gilani, S.A., Abbas, G., Ahmed, S., Khan, A.U., \& Choudhary, M.I. (2010). Antiglycation, antiplatelets aggregation, cytotoxic and phytotoxic activities of Nepeta suavis'. Lain. American Journal of Pharmacy, 29 (4): 573-8.

- Imran, I., Hussain, L., Ahmed, S., Rasool, N., Rasool, S., Abbas, G., Ali, M.Y. (2012). Antiplatelet activity of methanolic extract of Acacia leucophloea bark. Journal of Medicinal Plants Research, 6 (25): 4185.

- Kanjanapothi, D., Smitasiri, Y., Panthong, A., Taesotikul, T., Rathnapanone, V. (1981). Postcoital antifertility effect of Mentha arvensis. Contraception, 24: 559-567.

- Saeed, S.A., Khan, S.K., and Ahmed, S. (2003). The Inhibition of Prostaglandin Biosynthesis by Human Plasma and its Relationship to Albumin and Haptoglobin. Journal of Biological Sciences, 3 (12): 1188-1202.

- Saeed, S.A., Ahmed, S., Ali, A. (2003). A New Function of Human Haptoglobin: Endogenous inhibition of prostaglandin biosynthesis and its relation to hemoglobin binding. Journal of Medical Sciences, 3(5-6):344-357.

- Saeed, S.A., Rasheed, H., Ali, T.H., and Ahmed, S. (2004). Mechanisms of inhibitory actions of cyclooxygenase-2 inhibitors in human platelets. Journal of Biological Sciences, 4 (4): 515-520.

- Saeed, S.A., and Ahmed, S. (2005). New aspects of cyclooxygenase-2 inhibition in myocardial infarction and ischaemia. Research communications in molecular pathology and pharmacology, (117-118):167-78.

- Saeed, S.A., Motiwala, A., Qureshi, Z.U., Khan, R., Ali, A., Quadri J., and Ahmed, S. (2006). Mechanisms of platelet aggregation mediated by G-protein coupled receptors in human platelets. Journal of College of Physicians and Surgeons Pakistan, 16 (2): 167.

- Saeed, S.A., and Ahmed, S. (2006). Anti-ischemic Effects of Nimesulide, a Cyclooxygenase-2 Inhibitor on the Ischemic Model of Rabbit Induced by Isoproterenol. Archives of Pharmacal Research, 29(11):977-83. 
- $\quad$ Saeed, S.A., and Ahmed, S. (2006). Role of cyclooxygenase-2 in myocardial infarction and ischaemia. Journal of the College of Physicians and Surgeons-Pakistan, 16(5):324-8.

- Saeed, S.A., Connor, J.D., Quadri, J., Tasneem, S., Ahmed, S., Mesaik, M.A., Choudhary, M,I. (2007). Inhibitors of phosphatidylinositide 3-kinase: effects on reactive oxygen species and platelet aggregation. Pharmacol Reports, 59: 238 243.

- Saeed, S.A., Ahmad, N., Ahmed, S. (2007). Dual inhibition of cyclooxygenase and lipoxygenase by human haptoglobin: its polymorphism and relation to hemoglobin binding. Biochem. Biophys. Res. Commun, 353: 915 - 920.

- Satyavati, G.V., Gupta, A.K., Tandon, N. (1987). Medicinal Plants of India, vol. 2. ICMR, New Delhi.

- Shad, K.F., Aghazadeh, Y., Ahmed, S., Kress, Bodo. (2013). Peripheral markers of alzheimer's disease: Surveillance of white blood cells. Synapse, 67 (8), 541-3.

- Sharma, N., Jacob, D. (1996). Fertility suppression of the male mouse after administration of mint leaf extract. Phytotherapy Research, 10: 175-177.

- Sharma, N., and Jacob, D. (2002). Assessment of reversible contraceptive efficacy of methanol e xtract of Mentha arvensis L. leaves in male albino mice. J Ethnopharmacol. 80(1):9-13.

- Sylianco, C.Y.L., Blanco, F.R., Lim, C.M. (1986). Mutagenicity, clastogenicity and antimutagenicity of medicinal plant tablets produced by the NSTA pilot plant IV, Yerba buena tablets. Philipp. J. Sci. 115: 299-305.

- Steele, V.E., Holmes, C.A., Hawk, E.T., Kopelovich, L., Lubet, R.A., Crowell, J.A., Sigman, C.C., Kelloff, G.J. (1999) Lipoxygenase inhibitors as potential cancer chemopreventives. Cancer Epidemiol Biomarkers Prev. 8:467-83.

- Tang, D.G., Honn, K.V. (1997). Apoptosis of W256 carcinosarcoma cells of the monocytoid origin induced by NDGA involves lipid peroxidation and depletion of GSH: role of 12-lipoxygenase in regulating tumor cell survival. J Cell Physiol.172:155-70.

- Tyler, V. E. The honest herbal, 3rd ed.; Pharmaceutical Products Press: Binghamton, NY, 1993.

- Yamamoto, S., Suzuki, H., Nakamura, M., Ishimura, K. (1999). Arachidonate 12-lipoxygenase isozymes. Adv Exp Med Biol. 447:37-44.

- Zia-ul-Haq, M., Ahmed, S., Chivaro, E., Mehjabeen and Ahmed, S. (2010). Studies of oil from Cowpea (Vigna unguiculata (L.) Walp.) Cultivars commonly grown in Pakistan. Pakistan Journal of Botany, 42(2): 1333-1341.

- Zia-ul-Haq, M., Khan, B.A., Landa, P., Kutil, Z., Ahmed, S., Qayum, M., Ahmad, S. (2012). Platelet aggregation and anti-inflammatory effects of garden pea, Desi chickpea and Kabuli chickpea. Acta Poloniae Pharmaceutica, 69(4):707-11. 